\title{
Research on Virtual Reality Display of Ceramic Products
}

\author{
Chen $\mathrm{Bao}^{1}$, Bi Sheng ${ }^{1}$ \\ ${ }^{1}$ Jingdezhen Ceramic University, Jingdezhen, China
}

\begin{abstract}
With the synchronized development of computer and communication technology and the rapid rise and popularization of phase network technology, information application system has achieved a qualitative leap in breadth and depth. As a technical medium, virtual reality display provides a wedge machine for the development of different domains, including the ceramic domain. The employ of virtual reality technology to display ceramic products enable users to integrate into virtual products and virtual scenes, and interact with the exhibits in the scene at any time, which provides convenience for the display of ceramic products, thus promoting the development of ceramic industry and the spread of ceramic culture.
\end{abstract}

\section{Introduction}

Virtual display belongs to digital display, which comes into being and exists based on computer and network technology. It mainly uses virtual reality technology for three-dimensional display of objects, and it transforms the physical content of the display into symbols, images and other information ways. so that the object referred to by the symbol and the physical content of the image can be reproduced in the system of virtual technology. By employing virtual display technology and using computer simulation of three-dimensional environment, ceramic products make users have a sense of presence and can interact with the exhibits. This kind of active access to information can arouse great interest of users, increase the interaction, interest and entertainment between people and objects, and at the same time enhance the dissemination of cultural and artistic information.

\section{Introduction of Virtual Reality Technology}

Virtual reality technology is mainly based on the development of human-computer interaction technology. Generally speaking, the realization technology of virtual reality display mainly has the following two types: image-based panoramic technology and graphics-based virtual one. As a virtual reality technology, image-based panoramic technology mainly stitches the picture or video screen to realize the look around the scene and the three-dimensional drag display of the object. By taking one or more groups of photos of the real scene or object in the way of horizontal 360 degrees and vertical 360 degrees, and then seamlessly assembling them with professional software. And the employ of computer technology to acquire magnification, reduction, movement, commutation and other omni-directional interactive display of the real scene, in order to achieve the real effect of simulation and reproduction of the scene to express the complete surrounding environment information. The design path of graphics-based virtual display technology mainly uses the technology of producing and displaying three-dimensional graphics, generally with the assistance of professional modeling software (such as 3DSMAX, MAYA, etc.) to build three-dimensional scene and three-dimensional object model. This type of display can completely restore the scenes and objects in the real world, so the effect is rich, the display ability is powerful, and has good real-time interaction and a certain degree of autonomy.

\section{The Current Situation and Research Trend of Domestic Development.}

\subsection{The Current Situation of the Development of Virtual Reality Technology in China}

With the rapid improvement of computer system engineering and computer graphics technology, the state and government attach great importance to the research and improvement of virtual reality technology. Many domestic research institutions and universities have set up special research on the employ of virtual reality, and have acquired rich results. The first research on virtual reality technology in China is Beijing University of Aeronautics and Astronautics. They not only learn the fundamental knowledge, but also break through the representation and processing of the physical characteristics of objects in the virtual environment. The major achievements are highlighted in the hardware aspects such as visual interface, and develop the implementation path and related algorithms. At present, the related software of virtual production appears on the market one after another, and dozens of virtual reality technologies have been developed, such as VR-Platform, Virtools, Cult3D, 
EONStudio and so on. These softwares and technologies are widely used in various fields of the national economy and people's livelihood.

\subsection{Current Application Fields and Research Trends of Virtual Reality Technology}

Virtual reality technology has already been widely used, which plays an extremely significant role in urban planning, information data, medical field, real estate industry, e-commerce, network entertainment, network literature, distance education, information visualization and other fields. The use of virtual reality technology brings users a richer digital experience and is loved by more and more users. In the field of medicine, virtual reality technology is applied to the establishment of virtual human body model. through the use of tracking balls and sensory gloves, researchers can comprehend the internal organization of the human body in a more intuitive way. In the field of education, there are more products displayed by virtual reality technology in the market. They design APP, installed on a computer or mobile phone to scan pictures using a camera to generate three-dimensional animated models on the screen, and some products can interact richly. There is no doubt that this brings a lot of convenience and interest to our life. The adhibition of virtual reality technology also plays a active role in the cultural and entertainmental field. On the one hand, they reach users with the help of mobile Internet, and constantly use technologies such as big data to obtain user demand information and enhance the effect of user experience through sustaining progress; on the other hand, virtual reality technology gives cultural and entertainment products more space to play and produce, such as enabling users to cross into a very real historical environment. For instance, Zhejiang University opened a set of desktop virtual building real-time wandering system. The system employs layer superposition rendering technology and pre-blanking technology to achieve stereo vision, but also provides more convenient interactive tools, so that the real-time performance of the whole system and the realism of the picture have reached a very high level. Applications of virtual technology in the art field in the future has great potential for development. It not only plays an active role in establishing a sense of scene and atmosphere, but also perfectly presents the touch of musicians when playing a variety of musical instruments. Virtual reality technology is the result of intersection and mingle of many related disciplines. Its technical potential and application prospect are also very broad. Scientists will complete the improvement of human brain data reading technology in the near future, and scientific research predicts that eventually mankind will completely solve the problem of "human brain-computer interface". The virtual reality technology will lay more and more emphasis to the connection with users. Accordingly, from a technical point of view, we should strengthen the research on the technology based on multi-user virtual environment.

Virtual reality technology is full of vitality and has unlimited application prospects. However, at present, there are still many aspects that need to be broken through and problems to be solved. For example, in-depth research in the fields of new sensing and perceptual texture, new methods of geometry and modeling, high-speed graphics and image processing, high-performance computing, artificial intelligence, sociology, psychology and so on have made a qualitative leap to improve the interaction, fidelity and immersion of the system.

\section{The aims and contents of virtual display of ceramic products research}

\subsection{The aims of the research}

Through the exploration of the display characteristics and visual communication rules of different types of ceramic products, the virtual reality display of ceramic products intend to find out the best virtual expression suitable for different types of ceramic products, infuse the characteristics of ceramic culture and ceramic art with the support of virtual reality technology, realize omni-directional browsing and information interaction of the appearance, texture and scene of ceramic products, provide an interactive platform for the ceramic market, and promote and spread ceramic culture while improving economic benefits.

\subsection{Contents of the research}

In order to probe the virtual display of different types of ceramic products. Function is not only the essential attribute of the product, but also the significance and value of its existence as a product. At home and abroad, there are many classification methods of ceramic products, but it isn't a unified norm. In this paper, pottery products are roughly divided into five categories on the basis of its function: daily ceramics, art ceramics, industrial ceramics, building Yiwei classification raw ceramics, special ceramics and so on. In the products of daily ceramics, art ceramics and industrial ceramics are basically classified by their functions, because the emphasis of their respective functions and arts are different, therefore, the mode of expression chosen in the virtual display should also be different, in order to better express the information that the product needs to convey. In the display process of ceramic products, we are not only supposed to reflect the fundamental function, but also pay attention to the interaction between users and products. Based on this point, the functional experience element has become the main element in the ceramic product display. And combined with the artistic features of ceramic works to study the means of virtual display of ceramic products, using image panoramic display technology and VR display technology to make specific examples of the virtual display of different types of ceramic products, and realize the interactive design in the virtual software. finally, the research on the application of virtual reality display of ceramic products will be completed in the 
network or in the interactive projection of fixed occasions.

\subsection{Values of the research}

The research can create more commercial value by combining China's long ceramic culture and ceramic art with cutting-edge science and technology. Since the 21 century, the fast progress of information technology has brought about economic progress and the development of cultural industry, cultural and technological creation events have developed vigorously, and the environment for the connection of culture and science and technology has been formed. The cause of the integration of science and technology and culture has a bright future. The use of virtual reality technique, one of the forefronts of technology, to make a perfect cooperation between ancient culture and modern science is an extension of culture and art. It not only increases the spread path of ceramic culture and art, but also promotes the continuous development of science and technology. Ceramics show many aspects, such as national culture, social fashion, life customs and so on. It is the crystallization of the wisdom of human creation, which contains human culture. The technology of ancient Chinese traditional ceramics, through technical innovation, deduces the fashion of ancient Chinese civilization with a brand-new technology. It will also become a factor to enhance the potential value of ceramic products, which can make the audience identify with the products in spirit, so that developeing the economic and social effects of the products. Therefore, the use of virtual reality technology to convey the cultural connotation of ceramic products can not only derive more commercial value for the products, but also an effective way to use new technology and new cultural creation to tell good Chinese stories and promote the prosperity of the cultural industry in the new era.

\section{Conclusion}

As one of the four well-known ancient civilizations, China has made outstanding contributions to the civilization and evolution of human society, while the invention and development of ceramics is of more unique significance. From the Sui, Tang, the Five Dynasties, Song, Yuan, Ming and Qing dynasties to now, the craftsmanship and cultural connotations of ceramics have been constantly developing and evolving. They record and inherit the history and development of the nation, which can not be forgotten by future generations, and they are precious cultural heritages that need to be understood, loved and carefully protected by more people. Modern Chinese ceramics are highly respected because of the inheritance of traditional ceramic cultural spirit and artistic elements and the organic combination of high-technology tool. The use of virtual reality display technology in product development and promotion to develop ceramic products with high added value has injected new vitality into the ancient ceramic industry and told the story of China with the strength of science and technology.

\section{Acknowledgement}

Fund Project: Science and Technology Project of Jiangxi Provincial Department of Education (Project No. GJJ181533)

\section{REFERENCES}

1. Talking about the current situation and development trend of virtual reality technology, Yu Wenyan, Science and Technology Information,2012

2. Research on virtual display mode of ceramic products based on VR technology, Hu Lingli, 2012

3. Application of Virtual reality Technology in New Media display Design Li Shihao Mass Literature and Art,2016

4. Analysis of the present situation and trend of Virtual reality Technology 's development Wang Tingting's Digital Communication World,2017 